Global Journal of Pure and Applied Mathematics.

ISSN 0973-1768 Volume 16, Number 5 (2020), pp. 675-685

(C) Research India Publications

https://dx.doi.org/GJPAM/16.5.2020.675-685

\title{
Asymptotic of Second Order Polar Polynomials
}

\author{
Abdelhamid Rehouma, Tedjani Hadj Ammar and Abdelaziz Azeb Ahmed
}

Department of Mathematics, El Oued University, El Oued 39000, Algeria

\begin{abstract}
In this paper we derive useful results regarding the asymptotic properties of new set of monic polynomials primitives of orthogonal polynomials on the unit circle, called second order polar polynomials.
\end{abstract}

Keywords: Orthogonal polynomials, Polar polynomials, Asymptotic, Circle.

2010 Mathematics Subject Classification: Primary 05C38, 15A15; Secondary 05A $15,15 \mathrm{~A} 18$

\section{INTRODUCTION}

Let $\mu$ be a finite positive measure defined on the Borelian $\sigma$-algebra of $\mathbb{C}$ and concentrated on the unit circle $T=\{z,|z|=1\} . \mu$ is absolutely continuous with respect to the Lebesgue measure $d \theta$ on $[-\pi,+\pi]$, i.e

$$
d \mu(\theta)=\rho(\theta) d \theta, \quad \rho \geq 0, \quad \rho \in L^{1}([-\pi,+\pi], d \theta) .
$$

Let us consider $L_{n}(z)=z^{n}+\ldots .$. the $n$-th monic ( i.e its leading coefficient is equal to one) a monic orthogonal polynomial with respect to $\mu$, that is

$$
\frac{1}{2 \pi} \int_{0}^{2 \pi} L_{n}(z)(\bar{z})^{k} d \mu(\theta)=0, \quad k=0,1 \ldots ., n-1, \quad\left(z=e^{i \theta}\right) \text {. }
$$


Theses monic orthogonal polynomials are related by the following mutually equivalent recursions relations :

$$
L_{n}(z)=z L_{n-1}(z)+L_{n}(0) L_{n-1}^{*}(z) \quad \text { and } \quad L_{n}^{*}(z)=L_{n-1}^{*}(z)+\overline{L_{n}(0)} z L_{n-1}(z)
$$

where :

$$
L_{n}^{*}(z)=z^{n} \overline{L_{n}}\left(\frac{1}{z}\right), \quad(z \neq 0) .
$$

Let $\alpha$ be a fixed complex number, let us consider a monic polynomial $P_{n}(z)$ of degree $n$ and such that

$$
(n+1) L_{n}(z)=\left((z-\alpha) P_{n}(z)\right)^{\prime}=P_{n}(z)+(z-\alpha) P_{n}^{\prime}(z)
$$

$P_{n}$ is called the $n$-th polar polynomial of $L_{n}(z)$. For a fixed complex number $\alpha$, let us consider a monic polynomial $Q_{n}$ of degre $n$ such that

$$
\left((z-\alpha)^{2} Q_{n}(z)\right)^{\prime \prime}=(n+2)(n+1) L_{n}(z)
$$

$Q_{n}$ is called the $n$-second order polar polynomial of $L_{n}(z)$. Obviously

$$
\left((z-\alpha)^{2} Q_{n}(z)\right)^{\prime}=(n+2)(z-\alpha) P_{n}(z) .
$$

Note that $\Lambda(z)=(z-\alpha) P_{n}(z), \Pi(z)=(z-\alpha)^{2} Q_{n}(z)$ are respectively monic polynomials primitives of $(n+1) L_{n}(z)$, and $(n+2)(n+1) L_{n}(z)$ normalized respectively by $\Lambda(\alpha)=0$ and $\Pi(\alpha)=0$ and $\Pi^{\prime}(\alpha)=0 . P_{n}(z)$ is said to be the polar polynomial of $L_{n}(z)$ in the sense of Pijeira, (see [1]) for all details concerning polar polynomials of monic orthogonal polynomials with respect to a measure supported on un interval, in the case of $[-1,+1]$, instead of the unit circle $T=\{z,|z|=1\}$. One usually studies the asymptotics of $Q_{n}$ in one of four ways, the first is strong asymptotic

$$
\lim _{n \rightarrow \infty} \frac{Q_{n}^{\prime}(z)}{n L_{n}(z)}
$$

the second is root asymptotics :

$$
\lim _{n \longrightarrow \infty}\left|Q_{n}(z)\right|^{\frac{1}{n}}
$$

the third is ratio asymptotics :

$$
\lim _{n \rightarrow \infty} \frac{Q_{n+1}(z)}{Q_{n}(z)}
$$

and the fourth is Szegö asymptotics :

$$
\lim _{n \rightarrow \infty} \frac{Q_{n}(z)}{(\varphi(z))^{n}}, \quad \varphi(z) \text { is analytic on } \mathbb{C} \backslash \operatorname{ch}(\mu)
$$


where $\operatorname{ch}(\mu)$ denotes the convex hull of the support of the measure $\mu$.

A direct consequence of (2) and (4) is that

$$
\frac{1}{2 \pi} \int_{0}^{2 \pi}\left((z-\alpha)^{2} Q_{n}(z)\right)^{\prime \prime}(\bar{z})^{k} d \mu(\theta)=0, \quad k=0,1 \ldots n-1\left(z=e^{i \theta}\right) .
$$

This type of orthogonality relations generated by differential operators was intoduced initially by Aptekarev, Lagomazino, Marcelan, where the existence and uniqueness conditions for more general differential expressions were studied in detail. A similar study has been done by A. Fundora, H. Pijeira and W. Urbina [1], in the case of $[-1,+1]$. More information on the history and applications of this concept of polar may be found in $[1,10,12]$.

\subsection{Equilibrium measure}

In order to obtain the asymptotic behaviour of the 2-polar polynomials on the unit circle. We need some definitions and general results and lemmas that we will discuss in what follows

Lemma 1.1. $([1,6,14,17])$ Let $\Phi_{n}$ be a polynomial of degree $n$ with simple zeros $z_{n, 1}, z_{n, 2}, \ldots . z_{n, n}$. The normalised counting measure of the zeros of $\Phi_{n}$ is defined by

$$
\nu_{n}\left(\Phi_{n}\right)=\frac{1}{n} \sum_{k=1}^{n} \delta_{z_{n, k}}
$$

where $\delta_{z_{n, k}}$ is the Dirac measure with mass one at the point $z_{n, k}$, we get

$$
\lim _{n \longrightarrow \infty} \frac{\Phi_{n}^{\prime}(z)}{n \Phi_{n}(z)}=\lim _{n \longrightarrow \infty} \int \frac{d \nu_{n}(x)}{z-x}
$$

where $\lim _{n \longrightarrow \infty} \nu_{n}(x)=\omega_{\Delta}(x)$. in the sense of the weak $*$ topology $\nu_{n}$ is in this case the counting measure of the zeros of $\Phi_{n}$, where $\omega_{\Delta}$ is the equilibrium measure of $\Delta$.For $\Delta=[-1+1]$, the equilibrium measure is arcsin measure given by: if $B$ is Borel set in $[-1+1]$,

$$
\mu_{\Delta}(B)=\int_{B} \frac{\arcsin ^{\prime} x d x}{\pi}=\frac{1}{\pi} \int_{B} \frac{d x}{\sqrt{1-x^{2}}} .
$$

The normalised counting measure $\nu_{n}\left(P_{n}\right)$ converges to equilibrium measure arcsin measure in the sense of the weak *topolgy. Then

$$
\lim _{n \rightarrow \infty} \frac{\Phi_{n}^{\prime}(z)}{n \Phi_{n}(z)}=\int_{-1}^{1} \frac{d \omega_{\Delta}(x)}{z-x}=\frac{1}{\pi} \int_{-1}^{1} \frac{1}{z-x} \frac{d x}{\sqrt{1-x^{2}}} .
$$


Remark 1.2. In such a case (equilibrium measure $\omega_{\Delta}$ ). If $\nu_{n} \stackrel{*}{\longrightarrow} \omega_{\Delta}$, as $n \rightarrow \infty$,then

$$
\left\langle\nu_{n}(x), \frac{1}{z-x}\right\rangle \longrightarrow\left\langle\omega_{\Delta}(x), \frac{1}{z-x}\right\rangle .
$$

Lemma 1.3. ([1, 13]) Let $\Delta \subset \mathbb{C}$ be a compact set with empty interior and unbounded connected component with positive logarithmic capacity. If $\left\{\Phi_{n}(z)\right\}_{n=0}^{\infty}$ is a sequence of monic polynomials, $\operatorname{deg} \Phi_{n}(z)=n$, such that

$$
\limsup _{n \rightarrow \infty}\left(\left\|\Phi_{n}\right\|_{\Delta}^{\frac{1}{n}}\right) \leq \operatorname{Cap}(\Delta)
$$

then $\nu_{n}\left(\Phi_{n}\right) \stackrel{*}{\longrightarrow} \omega_{\Delta}$ here $\omega_{\Delta}$ is the equilibrium measure of $\Delta$.

Lemma 1.4. $([1,13,18])$ Let $\left\{\Phi_{n}(z)\right\}_{n=0}^{\infty}$ be a sequence of polynomials. Then for all $k \in \mathbb{Z}_{+}$

$$
\limsup _{n \rightarrow \infty} \sqrt[k]{\frac{\left\|\Phi_{n}^{(k)}\right\|_{\triangle}}{\left\|\Phi_{n}\right\|_{\triangle}}} \leq 1 .
$$

There is an interesting fact about Taylor's coefficients of polynomials and their zeros set and its local which based in the following Szegö's theorem (see [14, 15])

\subsection{Asymptotics of derivatives of monic orthogonal polynomials.}

There is a major theory for asymptotics of this type, with key initial advances due to Mate, Nevai, Rakhmanov, Totik and many later works (see [2]). In particular, Rakhmanov' theorem asserts that if $\mu>0$ a.e. on $[0,2 \pi]$, then we have this ratio asymptotic. Lubinsky ([8]) proved the following result asymptotic properties for monic orthogonal polynomials on the unit circle have been studied under different hypotheses. The Szegö's condition is satisfied. For instance,

$$
\int_{0}^{2 \pi} \log \mu^{\prime}>-\infty
$$

Asymptotics for derivatives of orthogonal polynomials have been established under various hypotheses by P.N evai and D.S.Lubinsky (see [8]).

Theorem 1.5. ([2, 3, 4]) Assuming the Szegö's condition and by the P. Nevai's condition hold, let $\left\{\varphi_{n}\right\}_{n=0,1,2 \ldots}$ denote the orthonormal polynomials for $\mu$, so that

$$
\frac{1}{2 \pi} \int_{0}^{2 \pi} \varphi_{n}(z) \overline{\varphi_{m}(z)} d \mu(\theta)=\delta_{m n} \quad\left(z=e^{i \theta}\right) .
$$


Let $m \geq 1$. Then

$$
\lim _{n \rightarrow \infty} \frac{z^{m} \varphi_{n}^{(m)}(z)}{n^{m} \varphi_{n}(z)}=1 \quad\left(z=e^{i \theta}\right)
$$

Theorem 1.6. ([8]) Lubinsky proved that for each $m \geq 1$ : if

$$
\lim _{n \rightarrow \infty} \frac{L_{n+1}(z)}{z L_{n}(z)}=1
$$

holds uniformly for compact subsets of $|z|=1$. Let $m \geq 1$. Then

$$
\lim _{n \rightarrow \infty} \frac{L_{n+1}^{(m)}(z)}{z L_{n}^{(m)}(z)}=1
$$

uniformly in $\{z,|z| \geq 1\}$.

Lemma 1.7. ([14, 15])(Szegô's theorem). Given the polynomials

$$
f_{n}(z)=\sum_{k=0}^{n} \alpha_{n k} C_{k}^{n} z^{k}, \alpha_{n n} \neq 0 \quad \text { and } g_{n}(z)=\sum_{k=0}^{n} \beta_{n k} C_{k}^{n} z^{k}, \quad \beta_{n n} \neq 0
$$

and

$$
h_{n}(z)=\sum_{k=0}^{n} \alpha_{n k} \beta_{n k} C_{k}^{n} z^{k} .
$$

If all the zeros of $f_{n}(z)$ lie in a closed disk $\bar{D}$ and $\lambda_{n, 1}, \lambda_{n, 2}, . . \lambda_{n, n}$ are the zeros of $g_{n}(z)$. Then every zero of $h_{n}(z)$ has the form $\lambda_{n, k} \gamma_{n, k}$, where $\gamma_{n, k} \in \bar{D}$.

\section{ASYMPTOTIC PROPRIETES OF THE SECOND ORDER POLAR POLYNOMIALS}

For the polar monic orthogonal polynomials and 2-polar polynomials and their zeros set and its local, there is an interesting fact about its Taylor's coefficients.

\subsection{Localization of zeros}

Our next propose is to prove that all the zeros of the polynomials of the two sequences $\left(P_{n}(z)\right)_{n=0,1,2 \ldots . .}$ and $\left(Q_{n}(z)\right)_{n=0,1,2 \ldots .}$ are contained in a disc which radius is independent of $n$.First, let us rewrite the polynomials $Q_{n}(z)$ and $P_{n}(z)$ in terms of $(z-\alpha)$, that is

$$
L_{n}(z)=\sum_{k=0}^{n} c_{n, k}(z-\alpha)^{k}, P_{n}(z)=\sum_{k=0}^{n} b_{n, k}(z-\alpha)^{k}, Q_{n}(z)=\sum_{k=0}^{n} a_{n, k}(z-\alpha)^{k} .
$$

It is well-known that the zeros of $L_{n}(z)$, (see ,Simon,[16] ) are contained in the unit closed disk $\bar{D}=\{z,|z| \leq 1\}$. 
Lemma 2.1. The coefficients $a_{n, k}$ of $Q_{n}$ and $b_{n, k}$ of $P_{n}$ and $c_{n, k}$ of $L_{n}$ in (12) are related by

$$
b_{n, k}=\frac{n+1}{k+1} c_{n, k}, a_{n, k}=\frac{n+2}{k+2} b_{n, k}
$$

i.e

$$
a_{n, k}=\frac{(n+2)(n+1)}{(k+2)(k+1)} c_{n, k} .
$$

Proof. Replacing (12) respectively in (3) and in (4) and (5).

Theorem 2.2. All the zeros of $P_{n}$ are contained in the closed disk $\bar{D}_{1}$, where

$$
\bar{D}_{1}=\{z \in \mathbb{C}:|z| \leq 2+3|\alpha|\} .
$$

All the zeros of $Q_{n}$ are contained in the closed disk $\bar{D}_{2}$, where

$$
\bar{D}_{2}=\{z \in \mathbb{C}:|z| \leq 2+5|\alpha|\} .
$$

Proof. Let us write, $w=z-\alpha$, hence

$$
f_{n}(w)=\sum_{k=0}^{n} c_{n, k} w^{k}=L_{n}(z) \quad \text { and } \quad h_{n}(w)=\sum_{k=0}^{n} \frac{n+1}{k+1} c_{n, k} w^{k}=P_{n}(z) .
$$

By Szegő's theorem,we get

$$
g_{n}(w)=\sum_{k=0}^{n} \frac{n+1}{k+1} C_{k}^{n} w^{k}
$$

we have

$$
g_{n}(w)=\frac{(1+w)^{n+1}-1}{w}=\frac{(z-\alpha+1)^{n+1}-1}{z-\alpha}, \quad(z \neq \alpha) .
$$

If $z_{n, 0}$ is a zero of $L_{n}$,it is well known that $\left|z_{n, 0}\right| \leq 1$, hence $w_{n, 0}=z_{n, 0}-\alpha$ is a zero of $f_{n}(w)$, lie in the closed disk $\bar{D}=\{|w+\alpha| \leq 1\}$. On the other hand,if $w_{n, 1}$ is a zero of $g_{n}(w)$ then: $\left|w_{n, 1}+1\right|=1$.Finally, by Lemma 4 , if $w_{n, 2}$ is a zero of $h_{n}(w)$, we have that $\left|w_{n, 2}\right|=\left|w_{n, 0} w_{n, 1}\right| \leq 2(1+|\alpha|)$.If $P_{n}\left(z_{n, 2}\right)=0$, then $z_{n, 2}=w_{n, 2}+\alpha$. Thus property (14) is confirmed.

Now in order to make (15), setting $w=z-\alpha$, hence

$$
F_{n}(w)=\sum_{k=0}^{n} b_{n, k} w^{k}=P_{n}(z) \quad H_{n}(w)=\sum_{k=0}^{n} \frac{n+2}{k+2} b_{n, k} w^{k}=Q_{n}(z)
$$

and

$$
G_{n}(w)=\sum_{k=0}^{n} \frac{n+2}{k+2} C_{k}^{n} w^{k}
$$


hence,

$$
\left(w^{2} G_{n}(w)\right)^{\prime}=\sum_{k=0}^{n}(n+2) C_{k}^{n} w^{k+1}=(n+2) w(1+w)^{n} .
$$

Integrating by parts both sides from 0 to $w$, we get

$$
w^{2} G_{n}(w)=\frac{n+2}{n+1} w(1+w)^{n+1}-\frac{1}{n+1}(1+w)^{n+2}
$$

i.e

$$
G_{n}(w)=\frac{(1+w)^{n+1}((n+1) w-1)}{(n+1) w^{2}} \quad,(w \neq 0)
$$

therefore,

$$
G_{n}(w)=\frac{(z-\alpha+1)^{n+1}((n+1) z-(n+1) \alpha-1)}{(n+1)(z-\alpha)^{2}}, \quad(z \neq \alpha) .
$$

If $w_{n, 3}$ is a zero of $G_{n}(w)$ then $\left|w_{n, 3}\right| \leq 1$.On the other hand, if $w_{n, 4}$ is a zero of $F_{n}(w)$ hence $w_{n, 4}=z_{n, 4}-\alpha$ where $P_{n}\left(z_{n, 4}\right)=0$, by (31) it is well known that $\left|z_{n, 4}\right| \leq 2+3|\alpha|$,then we have that $\left|w_{n, 4}+\alpha\right| \leq 2+3|\alpha|$. By Lemma 4, if $H_{n}\left(w_{n, 5}\right)=0$,we have that $\left|w_{n, 5}\right|=\left|w_{n, 3} w_{n, 4}\right| \leq 2+4|\alpha|$,thus property (15) is confirmed. The proof of the theorem is completed.

\subsection{Relative asymptotic of the of second order polar polynomials}

Relative asymptotic of the polar polynomials $\left\{P_{n}\right\}_{n=0,1,2 \ldots}$ with respect to the monic orthogonal polynomials $\left\{L_{n}\right\}_{n=0,1,2 \ldots}$ have been established under same hypotheses of P.Nevai and D.S.Lubinsky (see [8]) .

Theorem 2.3. ([12]) Let $\alpha$ be a fixed complex number. Let $P_{n}$ be the polar polynomial of $L_{n}$. Assume that

$$
\lim _{n \rightarrow \infty} \frac{L_{n+1}(z)}{z L_{n}(z)}=1
$$

uniformly for compact subsets of $\{|z|>1\}$. Then we have

$$
\lim _{n \rightarrow \infty} \frac{L_{n}(z)}{P_{n}(z)}=\frac{z-\alpha}{z}
$$

uniformly in $\{|z|>1\}$. And

$$
\lim _{n \rightarrow \infty} \frac{P_{n}^{\prime}(z)}{n P_{n}(z)}=\frac{1}{z}
$$

uniformly in $\{|z|>1\}$.In the same way,

$$
\lim _{n \rightarrow \infty} \frac{P_{n+1}(z)}{z P_{n}(z)}=1
$$

uniformly in $\{|z|>1\}$. 
Proof. see ([12]).

The main result is the following theorem :

Theorem 2.4. With the above assumptions, let $\alpha$ be a fixed complex number. Let $Q_{n}$ be the 2-polar polynomial of $L_{n}$. Assume that

$$
\lim _{n \rightarrow \infty} \frac{L_{n+1}(z)}{z L_{n}(z)}=1
$$

then

$$
\lim _{n \longrightarrow \infty} \frac{Q_{n+1}(z)}{z Q_{n}(z)}=1
$$

uniformly in $\{|z|>1\}$.it holds

$$
\lim _{n \longrightarrow \infty} \frac{Q_{n}^{\prime}(z)}{n Q_{n}(z)}=\frac{1}{z}
$$

uniformly in $\{|z|>1\}$.

Proof. By (5),

$$
(z-\alpha)^{2} Q_{n}^{\prime}(z)+2(z-\alpha) Q_{n}(z)=(n+2)(z-\alpha) P_{n}(z), \quad(z \neq \alpha) .
$$

Then

$$
\lim _{n \longrightarrow \infty} \frac{P_{n}(z)}{(z-\alpha) Q_{n}(z)}=\lim _{n \longrightarrow \infty} \frac{Q_{n}^{\prime}(z)}{n Q_{n}(z)}
$$

If $z \neq \alpha$, we write

$$
\frac{d}{d z} \log (z-\alpha)^{2} Q_{n}(z)=\frac{\left((z-\alpha)^{2} Q_{n}(z)\right)^{\prime}}{(z-\alpha)^{2} Q_{n}(z)} \quad(z \neq \alpha)
$$

hence

$$
\frac{d}{d z} \log (z-\alpha)^{2} Q_{n}(z)=(n+2) \frac{P_{n}(z)}{(z-\alpha) Q_{n}(z)} \quad(z \neq \alpha) .
$$

By Integration in both hand sides of this equality from fixed point $z_{1}$ to $z$, we get

$$
\frac{(z-\alpha)^{2} Q_{n}(z)}{\left(z_{1}-\alpha\right)^{2} Q_{n}\left(z_{1}\right)}=\exp \left((n+2) \int_{z_{1}}^{z} \frac{P_{n}(t)}{(t-\alpha) Q_{n}(t)} d t\right) \quad(t \neq \alpha)
$$

and

$$
Q_{n}(z)=\left(\frac{z_{1}-\alpha}{z-\alpha}\right)^{2} Q_{n}\left(z_{1}\right) \exp \left((n+2) \int_{z_{1}}^{z} \frac{P_{n}(t)}{(t-\alpha) Q_{n}(t)} d t\right)
$$


Taking into account this expression (23) and (22) reads .

$$
\lim _{n \longrightarrow \infty} \frac{Q_{n+1}(z)}{Q_{n}(z)}\left(\frac{Q_{n+1}\left(z_{1}\right)}{Q_{n}\left(z_{1}\right)}\right)^{-1}=\exp \left(\lim _{n \longrightarrow \infty} \int_{z_{1}}^{z} \frac{P_{n}(t)}{(t-\alpha) Q_{n}(t)} d t\right) .
$$

Thus, we have

$$
\lim _{n \longrightarrow \infty} \frac{Q_{n+1}(z)}{Q_{n}(z)}\left(\frac{Q_{n+1}\left(z_{1}\right)}{Q_{n}\left(z_{1}\right)}\right)^{-1}=\exp \Lambda(z)
$$

where

$$
\Lambda(z)=\int_{z_{1}}^{z} M(t) d t
$$

and

$$
M(z)=\lim _{n \longrightarrow \infty} \frac{Q_{n}^{\prime}(z)}{n Q_{n}(z)} .
$$

Which yields the proof of the existence of $M(z)$. It is easy to conclude that

$$
\lim _{n \rightarrow \infty} \frac{Q_{n+1}(z)}{Q_{n}(z)}=\lim _{n \rightarrow \infty} \frac{P_{n+1}(z)}{P_{n}(z)}=z
$$

threfore,

$$
\exp \Lambda(z)=\frac{z}{z_{1}}, \quad\left(z_{1} \text { is fixed point }\right)
$$

implies

$$
M(z)=\Lambda^{\prime}(z)=\frac{1}{z} .
$$

The proof of proprietes (19),(20) may now be completed.

Remark 2.5. All the zeros of $Q_{n}$ are contained in the closed disk $\bar{D}_{2}$, where

$$
\bar{D}_{3}=\{z \in \mathbb{C}:|z| \leq 1+2|\alpha|\} .
$$

To make (24), setting $w=z-\alpha$,by (14) :

$$
f_{n}(w)=\sum_{k=0}^{n} c_{n, k} w^{k}=L_{n}(z), T_{n}(w)=\sum_{k=0}^{n} \frac{(n+2)(n+1)}{(k+2)(k+1)} c_{n, k} w^{k}=Q_{n}(z)
$$

and

$$
R_{n}(w)=\sum_{k=0}^{n} \frac{(n+2)(n+1)}{(k+2)(k+1)} C_{k}^{n} w^{k}, \quad w \neq 0
$$

hence,

$$
\left(w^{2} R_{n}(w)\right)^{\prime \prime}=(n+2)(n+1) \sum_{k=0}^{n} C_{k}^{n} w^{k}=(n+2)(n+1)(1+w)^{n}
$$




$$
R_{n}(w)=\frac{(1+w)^{n+2}}{w^{2}}=\frac{(z-\alpha+1)^{n+2}}{(z-\alpha)^{2}}, \quad z \neq \alpha .
$$

If $s_{n, 3}$ is a zero of $R_{n}(w)$ then $\left|s_{n, 3}\right| \leq 1$.On the other hand, if $s_{n, 4}$ is a zero of $f_{n}(w)$ hence $s_{n, 4}=z_{n, 4}-\alpha$ where $L_{n}\left(z_{n, 4}\right)=0$, it is well known that $\left|z_{n, 4}\right| \leq 1$, then we have that $\left|s_{n, 4}+\alpha\right| \leq 1$, by Lemma 4,if $T_{n}\left(s_{n, 5}\right)=0$,since $s_{n, 5}=z_{n, 5}-\alpha$, where $Q_{n}\left(z_{n, 5}\right)=0$, we have that $\left|s_{n, 5}\right|=\left|s_{n, 3} s_{n, 4}\right| \leq\left|s_{n, 3}\right|\left|s_{n, 4}+\alpha-\alpha\right| \leq 1+|\alpha|$, the property (15) is confirmed.

Remark 2.6. Since,by (22)

$$
\lim _{n \longrightarrow \infty}\left(\frac{P_{n}(z)}{Q_{n}(z)}\right)^{\prime} \lim _{n \rightarrow \infty} \frac{P_{n}(z)}{Q_{n}(z)}\left(\frac{P_{n}^{\prime}(z)}{Q_{n}(z)}-\frac{Q_{n}^{\prime}(z)}{Q_{n}(z)}\right)
$$

imply,

$$
\lim _{n \rightarrow \infty} \frac{Q_{n}^{\prime}(z)}{n Q_{n}(z)}=\lim _{n \rightarrow \infty} \frac{P_{n}^{\prime}(z)}{n P_{n}(z)}=\frac{1}{z}
$$

\section{REFERENCES}

[1] A. Fundora, H. Pijeira and W. Urbina, Asymptotics behavior of orthogonal polynomials primitives. Margarita mathematica en memoira de José Javier Guadalupe Hernández, Servicio de Publicacone de la Univ.de la Rioja,Logrõno, Spain (2001) 626-632.

[2] E. A. Rahmanov, On the asymptotics of the ratio of orthogonal polynomials .Math. USSR Sbornik 32(2) (1977). 199-213.

[3] E. A. Rahmanov, On the asymptotics of the ratio of orthogonal polynomials. 11 .Math. USSR Sbornik 46(1) (1983). 105-117.

[4] P. Nevai Géza Reud. Orthogonal Polynomialsand Christoffel functions. A case study. J. of Appr. Th. 46 (1986). 3-167.

[5] P. Nevai, Orthogonal polynomials, measures and recurrence relations on the unit circle. Transact. of the Amer. Math.Soc. 300 (1) 1987. 175-189.

[6] P. Nevai and V. TOTIK, Orthogonal polynomials and their zeros". Acta Scient. Math.(Szeged). 53(1-2) (1989). 99-104.

[7] J. Alves ,D. Castonga and T.Brustle, A polynomial recognition of unit forms using graph-based strategies,Discrete Applied Mathematics.Volume 253, 30 January 2019, 61-72.

[8] D.S. Lubinsky, On Asymptotics of Derivatives of Orthogonal Polynomials on the Unit Circle.J-Approx.theory 145(2007), 122-127 
[9] A. AZIZ ans B.A.ZERGAR, On the Zeros of Polynomials. Proc.Indian acad .Sci.Mat Sci,vol 106, No 2 , May 1996, 127-132.

[10] H.Pijeira, Y .Cabrera, Y .Jose.Bello, W. Cruz and R.Urbina, On Polar Legendre polynomials, Rocky Mountain Journal of Mathematics,RMJ (2008), vol 38,1-10.

[11] G. Kresin, An Extremal Problem for Integrals on a Measure Space with Abstract Parameters,Complex Analysis and Operator Theory .Vol. 11 issue.7 ,October 2017. 1477-1490.

[12] A. Rehouma, YA.Laskri and R.Benzine, Asymptotic Properties of Polar Polynomials,Applied Mathematical Sciences,(AMS) Vol. 8, no. 14. (2014), 685 $-691$.

[13] H.N. Mhaskar and E.B. Saff, On the distribution of zeros of polynomials orthogonal on the unit circle.Journal of appproximation theory .Vol 63,N 1,(1990).

[14] G. Szegő, Bemerkungen zu einen Satz von J. H. Grace, Uber die Wurzeln algebraischer Gleighungen, Math. Z. 13 (1922), 28-55.

[15] G. Szegô, Orthogonal Polynomials .Amer. Math. Soc. Coll. Publ. vol 23 (4th edition). Providence. (1975).

[16] B. Simon, Orthogonal polynomials on the unit circle,part 1:Classical theory ,AMS Colloquium Series,Americain Mathematical Society,Providence,RI, (2005).

[17] YA.L. Geronimos, Polynomials orthogonal on a circle and their applications, Series and Approximations, Amer. Math. Soc. Transl., series 1, vol. 3, Providence, Rhode Island,(1962) 1-78.

[18] H. Stahl and V. Totik, General Orthogonal Polynomials, Cambridge University Press, Cambridge,(1992).

[19] L. Garza,F. MarcellARCELLán, Verblunsky Parameters and Linear Spectral Transformations, Methods and Applications of Analysis 16 (2009), no. 1, 69-86. 
